\title{
Favorable outcomes of COVID-19 in recipients of hematopoietic cell transplantation
}

\author{
Gunjan L. Shah,, ${ }^{1,2}$ Susan DeWolf, ${ }^{1}$ Yeon Joo Lee, ${ }^{2,3}$ Roni Tamari, ${ }^{1,2}$ Parastoo B. Dahi, ${ }^{1,2}$ Jessica A. Lavery, ${ }^{4}$ Josel Ruiz, ${ }^{1}$ \\ Sean M. Devlin, ${ }^{4}$ Christina Cho, ${ }^{1,2}$ Jonathan U. Peled, ${ }^{1,2}$ Ioannis Politikos, ${ }^{1,2}$ Michael Scordo, ${ }^{1,2}$ N. Esther Babady, ${ }^{5}$ Tania Jain, ${ }^{1}$ \\ Santosha Vardhana, ${ }^{2,6}$ Anthony Daniyan, ${ }^{2,7}$ Craig S. Sauter, ${ }^{1,2}$ Juliet N. Barker, ${ }^{1,2}$ Sergio A. Giralt, ${ }^{1,2}$ Cheryl Goss, ${ }^{8}$ Peter Maslak, ${ }^{9}$ \\ Tobias M. Hohl, ${ }^{2,3}$ Mini Kamboj, ${ }^{2,3}$ Lakshmi Ramanathan, ${ }^{10}$ Marcel R.M. van den Brink, ${ }^{1,2}$ Esperanza Papadopoulos, ${ }^{1,2}$ \\ Genovefa Papanicolaou, ${ }^{2,3}$ and Miguel-Angel Perales ${ }^{1,2}$
}

'Adult Bone Marrow Transplant Service, Department of Medicine, Memorial Sloan Kettering Cancer Center, New York, New York, USA. ²Department of Medicine, Weill Cornell Medical College, New York, New York, USA. ${ }^{3}$ Infectious Disease Service, Department of Medicine; ${ }^{4}$ Department of Epidemiology and Biostatistics; ${ }^{5}$ Clinical Microbiology Service, Department of Laboratory Medicine; ${ }^{6}$ Lymphoma Service and 'Leukemia Service, Department of Medicine; and ${ }^{8}$ Transfusion Medicine Service, ${ }^{9}$ Cellular Immunology Laboratory, and ${ }^{10} \mathrm{Cl}$ linical Chemistry Service, Department of Laboratory Medicine, Memorial Sloan Kettering Cancer Center, New York, New York, USA.

BACKGROUND. Understanding outcomes and immunologic characteristics of cellular therapy recipients with SARS-CoV-2 is critical to performing these potentially life-saving therapies in the COVID-19 era. In this study of recipients of allogeneic (Allo) and autologous (Auto) hematopoietic cell transplant and CD19-directed chimeric antigen receptor T cell (CAR T) therapy at Memorial Sloan Kettering Cancer Center, we aimed to identify clinical variables associated with COVID-19 severity and assess lymphocyte populations.

METHODS. We retrospectively investigated patients diagnosed between March 15, 2020, and May 7, 2020. In a subset of patients, lymphocyte immunophenotyping, quantitative real-time PCR from nasopharyngeal swabs, and SARS-CoV-2 antibody status were available.

RESULTS. We identified 77 patients with SARS-CoV-2 who were recipients of cellular therapy (Allo, 35; Auto, 37; CAR T, 5; median time from cellular therapy, 782 days; IQR, 354-1611 days). Overall survival at 30 days was $78 \%$. Clinical variables significantly associated with the composite endpoint of nonrebreather or higher oxygen requirement and death ( $n$ events $=25$ of 77 ) included number of comorbidities (HR 5.41, $P=\mathbf{0 . 0 0 4}$ ), infiltrates (HR 3.08, $P=0.032$ ), and neutropenia (HR 1.15, $P=0.04$ ). Worsening graft-versus-host disease was not identified among Allo recipients. Immune profiling revealed reductions and rapid recovery in lymphocyte populations across lymphocyte subsets. Antibody responses were seen in a subset of patients.

CONCLUSION. In this series of Allo, Auto, and CAR T recipients, we report overall favorable clinical outcomes for patients with COVID-19 without active malignancy and provide preliminary insights into the lymphocyte populations that are key for the antiviral response and immune reconstitution.

FUNDING. NIH grant P01 CA23766 and NIH/National Cancer Institute grant P30 CA008748.

Authorship note: GLS and SD contributed equally to this work.

Conflict of interest: GLS reports research funding from Janssen and Amgen. YJL receives support for conducting industry-sponsored trials from Astellas Pharma and Ansun BioPharma. PBD served on an advisory board for Kite, a Gilead company. JUP reports research funding, intellectual property fees, and travel reimbursement from Seres Therapeutics and consulting fees from DaVolterra. IP reports research funding from Merck and serves on a Data and Safety Monitoring Board for ExCellThera. MS has served as a paid consultant for McKinsey \& Company, Angiocrine Bioscience Inc., and Omeros Corporation. He reports research funding from Angiocrine Bioscience Inc. He has served on an ad hoc advisory board for Kite, a Gilead company. TJ reports consultancy honoraria from Takeda Oncology and has served on an advisory board for CareDx. SV reports personal fees from Immunai and personal fees from ADC Therapeutics; in addition, SV has a pending patent (PCT/US19/27610). CSS has served as a paid consultant on advisory boards for Juno Therapeutics; Sanofi-Genzyme; Spectrum Pharmaceuticals; Novartis, Genmab; Precision Biosciences; Kite, a Gilead company; Celgene; Gamida Cell; and GlaxoSmithKline. He reports research funding from Juno Therapeutics, Celgene, Precision Biosciences, and Sanofi-Genzyme. JNB reports research funding from Angiocrine Bioscience, Gamida Cell, and Merck. SAG has consulted for and received research funding from Amgen, Actinium, Celgene, Johnson \& Johnson, Bristol-Myers Squibb, Sanofi, Pfizer, and Takeda and has received research funding from Miltenyi Biotec. SAG has consulted for Jazz Pharmaceuticals; GlaxoSmithKline; Novartis; Kite, a Gilead company; and Spectrum Pharmaceuticals. TMH has participated in scientific advisory boards for Merck and Partner Therapeutics. MRMVDB has received research support from Seres Therapeutics; has consulted, received honorarium from, or participated in advisory boards for Seres Therapeutics, Forty-Seven Inc., Magenta, Juno Therapeutics, Rheos, WindMIL Therapeutics, Novartis, Evelo, Jazz Pharmaceuticals, Therakos, Amgen, Magenta Therapeutics, Merck, Acute Leukemia Forum, and DKMS Medical Council (board); has IP licensing with Seres Therapeutics and Juno Therapeutics; and stock options from Smart Immune. MAP has served on advisory boards for MolMed, NexImmune, Medigene, and Servier; has received honoraria and served on advisory boards for Abbvie, Bellicum, Bristol-Meyers Squibb, Nektar Therapeutics, Novartis, Omeros, and Takeda; has consulted for and received honoraria from Merck; and has received research funding from Incyte, Miltenyi Biotec, Kite, a Gilead company. Copyright: () 2020, American Society for Clinical Investigation.

Submitted: June 29, 2020; Accepted: September 2, 2020; Published: November 16, 2020.

Reference information: / Clin Invest. 2020;130(12):6656-6667. https://doi.org/10.1172/JCI141777. 


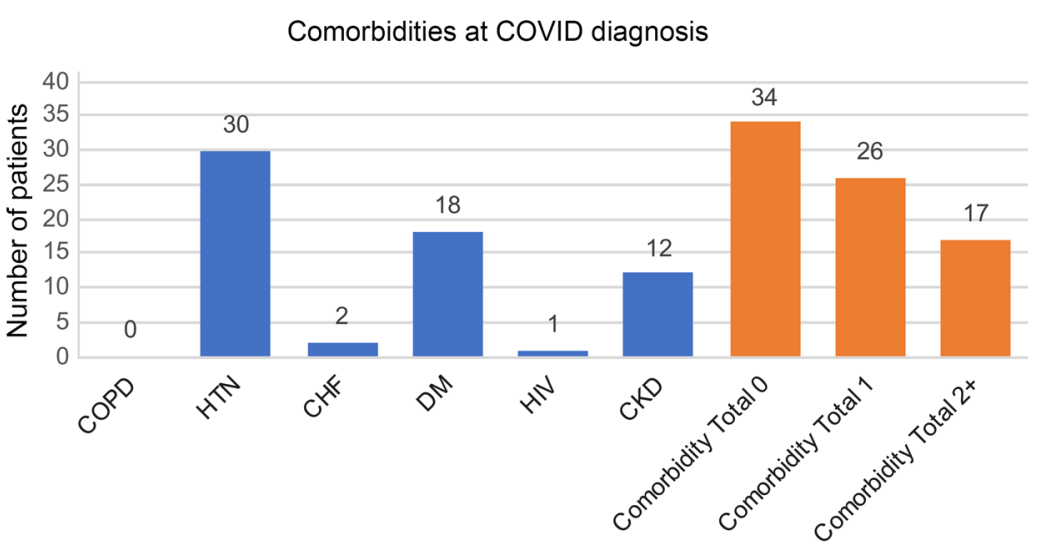

Figure 1. Comorbidities at COVID-19 diagnosis. Seventyseven patients (Allo $n=35$, Auto $n=37$, CAR T $n=5$ ). COPD, chronic obstructive pulmonary disease; HTN, hypertension; CHF, congestive heart failure; DM, diabetes mellitus; CKD, chronic kidney disease.

\section{Introduction}

As of June 2, 2020, there were over 1.8 million confirmed cases of coronavirus disease 2019 (COVID-19), which is caused by the severe acute respiratory syndrome coronavirus-2 (SARS-CoV-2), in the United States, with more than 16,000 deaths in New York City (1). The vulnerability of patients with significant comorbidities became evident early in this public health crisis. Patients with cancer have been considered as potentially one of the most at-risk groups due their immunocompromised state related to their underlying malignancy and associated treatments; however, studies primarily included patients with solid tumors (2). More recent studies focused on patients with hematologic malignancy confirmed that previously identified risk factors for disease severity also hold true for these patients (3-7).

$\mathrm{T}$ cells are the key mediators of antiviral immune responses, and studies of lymphocytes in patients with COVID-19 are beginning to emerge (8). Lymphopenia is the hallmark of severe COVID-19 presentation (9), and studies from small series of patients suggest this affects T cells, B cells, and NK cells (10-13). Recipients of cellular therapies, including allogeneic hematopoietic cell transplantation (Allo), autologous hematopoietic cell transplantation (Auto), and CD19-directed chimeric antigen receptor $\mathrm{T}$ cell (CAR T) therapy, constitute a unique population of patients with hematologic malignancies, due to their immune dysregulation and prolonged timeline for immune reconstitution.

In this study, we sought to characterize the clinical course of patients with hematologic malignancies who previously received Allo, Auto, or CAR T therapy and evaluated changes in lymphocyte and $\mathrm{T}$ cell subsets during SARS-CoV-2 infection at Memorial Sloan Kettering Cancer Center (MSKCC). With need to conserve hospital resources and concern for patient safety, centers performed only emergent transplants under guidelines that suggest delaying elective transplants and cellular therapies during the pandemic. Here, we describe potential risk factors for severe disease in this immunocompromised population to allow for mitigation and treatment of COVID-19 and to guide transplant centers as they resume these potentially life-saving treatments based on local conditions.

\section{Results}

\section{Demographics, disease, and treatment characteristics}

Between March 11, 2020, and May 7, 2020, 77 patients (Allo, $n=35$; Auto, $n=37$; CAR T, $n=5$ ) met criteria for diagnosis of
COVID-19, with median follow-up in surviving patients of 23 days (IQR, 14-35 days). The median age at COVID-19 diagnosis was 62 years (range, $25-78$ years), with $17 \%$ over the age of 70 years and $64 \%$ male sex (Table 1). Median time from most recent cell therapy was 782 days (IQR, 354-1611 days). All CAR T recipients received FDA-approved commercial products with $80 \%$ axicabtagene ciloleucel. At time of COVID-19 diagnosis, 17\% of Allo recipients had active graft-versus-host disease (GVHD), which did not worsen during their course. No patients had a new diagnosis of GVHD during their COVID-19 treatment.

Most patients had never smoked (66\%) or vaped (96\%). The median BMI was $27.4 \mathrm{~kg} / \mathrm{m}^{2}$ (IQR, $24.1-30.6 \mathrm{~kg} / \mathrm{m}^{2}$ ). At the time of COVID-19 diagnosis, $22 \%$ of patients had 2 comorbidities when considering hypertension, congestive heart failure, chronic obstructive pulmonary disease, diabetes mellitus, HIV, and chronic kidney disease, while $44 \%$ had none of these issues (Figure 1). Patients were on aspirin (26\%); immunomodulatory agents (lenalidomide/pomalidomide, 23\%); GVHD immunosuppressive agents (tacrolimus, cyclosporine, mycophenolate mofetil, and/or ruxolitinib, 18\%); steroids (13\%); angiotensin-convertingenzyme inhibitors/angiotensin receptor blockers (7\%); and anticoagulation medications (5\%). No patients were on BTK inhibitors at the time of COVID-19 diagnosis. Thirteen percent of patients received intravenous immunoglobulin within 3 months prior to COVID-19 diagnosis.

Regarding the status of the hematologic malignancy, 25\% had relapse or progression of disease after Allo, Auto, or CAR T. At the time of COVID-19 diagnosis, the most recent disease status was in remission not on treatment, in remission on consolidation or maintenance treatment, stable disease but not in remission, or relapsed/refractory disease in $48 \%, 22 \%, 14 \%$, and $16 \%$ of patients, respectively. As most patients were in remission or on maintenance, $62 \%$ did not have any changes in treatment plan at time of diagnosis, but treatment was delayed or permanently discontinued in $31 \%$ and $3 \%$, respectively.

\section{Symptoms and clinical course}

Clinical presentation. Seventy-four patients had a positive nasopharyngeal swab (NPS) for SARS-CoV-2 RNA (25\% tested outside MSKCC), with 3 patients having presumed disease and $45 \%$ having a known positive contact. Symptoms at diagnosis included cough (65\%), fever (58\%), fatigue (39\%), shortness of breath 


\section{Table 1. Patient characteristics}

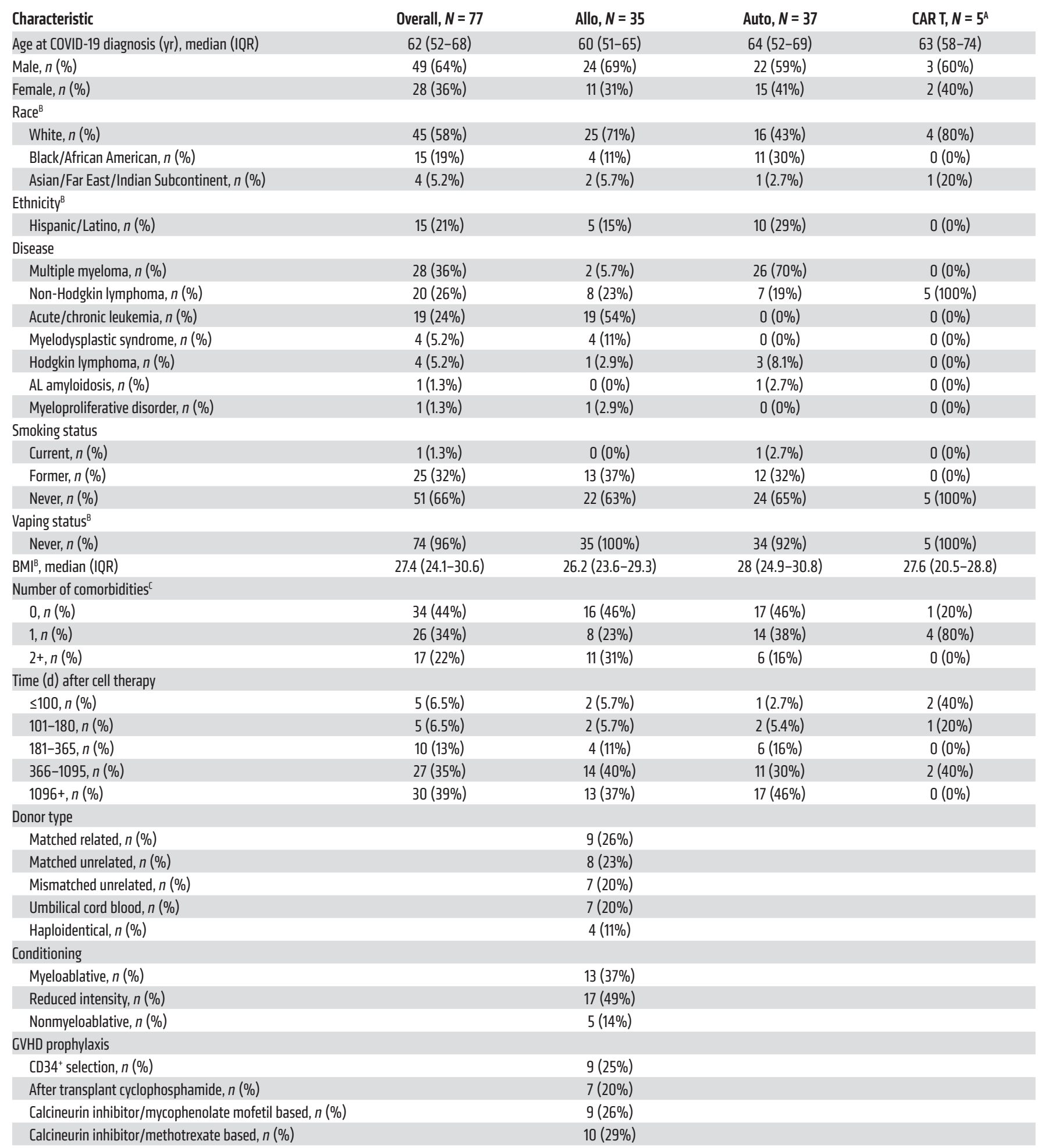

AOne patient who had a CAR T had a prior Auto. BUnknown race ( $n=2$ Allo; 13,2 , and 9 patients in the Overall, Allo, and Auto groups belonged to other racial groups), ethnicity ( $n=8 ; 4$ Allo, 2 Auto, 2 CAR T), vaping status $(n=1)$, BMI $(N=6)$. 'Comorbidities include hypertension, congestive heart failure, chronic obstructive pulmonary disease, diabetes mellitus, HIV, and chronic kidney disease. 


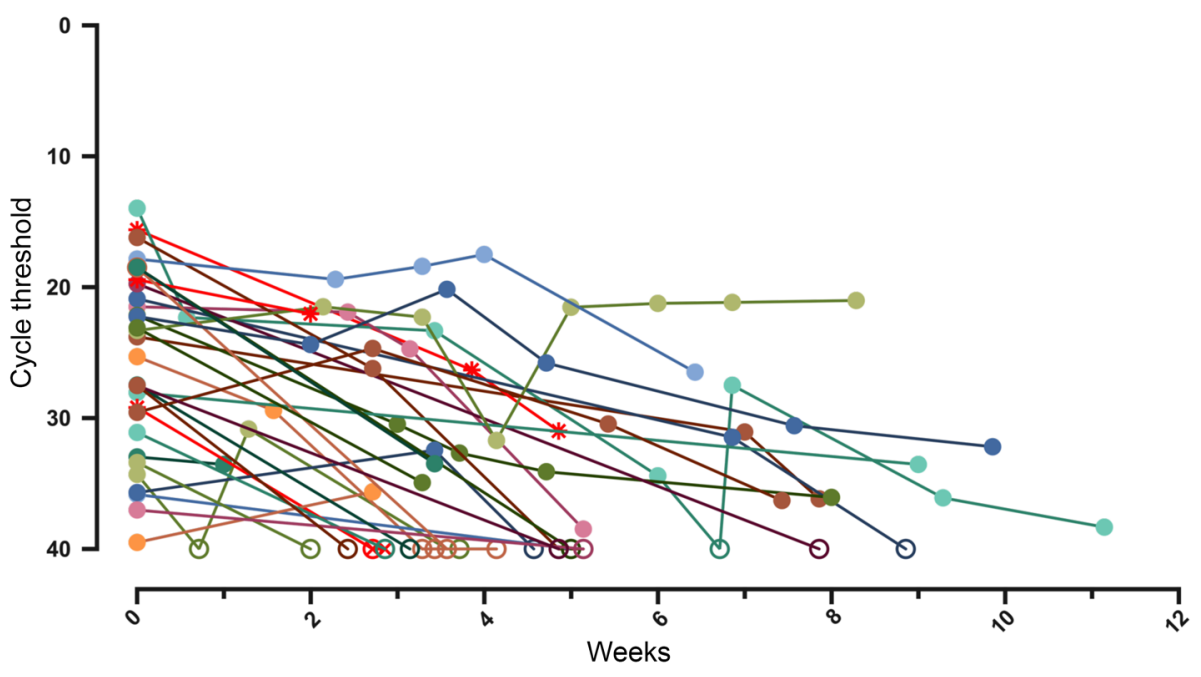

Figure 2. Monitoring patients with SARSCoV-2 over time. Cycle threshold data over time for patients with 2 or more PCR swabs $(n=31)$. All negative values were given a value of 40 ( $C t \geq 40=$ negative test at MSKCC, indicated with open circle). Red asterisks indicate that the patient is deceased.
(30\%), myalgias (27\%), headache (16\%), nausea/vomiting (10\%), anosmia (9\%), rhinorrhea (8\%), confusion (8\%), diarrhea (7\%), and diaphoresis (4\%). At time of initial positive NPS, oxygen saturation was checked in 43 patients (56\%) and was below $90 \%$ in $21 \%$. Fifty percent of patients had imaging done, with $64 \%$ of those studies revealing an infiltrate.

Laboratory data. Laboratory tests were performed in $65 \%$ of patients at the time of COVID-19 diagnosis. The median neutrophil count was $3.2 \mathrm{k} / \mu \mathrm{L}$ (IQR, 1.7-5 k/ $\mu \mathrm{L}$ ), $3.4 \mathrm{k} / \mu \mathrm{L}$ (IQR, 1.9-6.2 $\mathrm{k} / \mu \mathrm{L}$ ), $2.8 \mathrm{k} / \mu \mathrm{L}$ (IQR, 1.5-4.4 k/ $\mu \mathrm{L}$ ), and $3.6 \mathrm{k} / \mu \mathrm{L}$ (IQR, 2.9-4.3 $\mathrm{k} / \mu \mathrm{L}$ ) for all patients and those receiving Allo, Auto, and CAR T, respectively. Lymphopenia was common, with the median absolute lymphocyte count (ALC) $0.9 \mathrm{k} / \mu \mathrm{L}$ (IQR, 0.5-1.5 k/ $\mu \mathrm{L}$ ), 0.9 $\mathrm{k} / \mu \mathrm{L}$ (IQR, 0.6-2 k/ $\mu \mathrm{L}$ ), $0.9 \mathrm{k} / \mu \mathrm{L}$ (IQR, $0.5-1.3 \mathrm{k} / \mu \mathrm{L}$ ), $0.3 \mathrm{k} / \mu \mathrm{L}$ (IQR, 0.3-0.4 k/ $\mu \mathrm{L}$ ) for all patients and those receiving Allo, Allo, Auto, and CAR T, respectively. Overall, the median neutrophil/ lymphocyte ratio was 3.55 (range, 0.67-60). Renal and hepatic function was mostly not affected. Additional laboratory values at time of positive NPS, time of admission, and maximums throughout COVID-19 course are shown in Supplemental Table 1 (supplemental material available online with this article; https://doi. org/10.1172/JCI141777DS1). Median maximum values of inflammatory markers included $1396 \mathrm{ng} / \mathrm{mL}$ ferritin (IQR, 277-4305 $\mathrm{ng} / \mathrm{mL}, n=30$ ), $16.9 \mathrm{mg} / \mathrm{dL}$ C-reactive protein (IQR, 10.1-26.4 $\mathrm{mg} / \mathrm{dL}, n=31$ ), and (pretocilizumab in those that received it) 93.5 $\mathrm{pg} / \mathrm{mL}$ IL-6 (IQR, 34.3-231 pg/mL, $n=30$ ).

Cycle threshold $(\mathrm{Ct})$ is a semiquantitative estimate of the viral load on a NPS and was available for $68 \%$ of patients, with median Ct for N2 (a region of the nucleocapsid gene) on diagnostic NPS of 22.65 (IQR, 19.53-29.18). Routine swabbing until negativity was not done; however, of the $58 \%$ of patients with serial testing, a median of 2 NPS (IQR, 2-3.5) were done, with $52 \%$ being negative on the most recent NPS and median time to negativity of 28 days (IQR, 22-35). In those in which the most recent NPS was still positive, median time from initial positive to most recent positive was 44 days (IQR, 23-57 days). Ct values trended upward overtime in most but not all patients during the study period (Figure 2).

COVID-19-directed treatments. COVID-19-directed treatment was given to $47 \%$ of patients overall, with 1 of 3 of patients receiv- ing treatment on a clinical trial. The most common treatments included hydroxychloroquine (32\%) started a median of 1 day after COVID-19 diagnosis (IQR, 1-2 days), azithromycin (25\%) at 1 day (IQR, 0-2 days ), methylprednisolone (18\%) at 6 days (IQR, 4-11 days), convalescent plasma (16\%) at 10 days (IQR, 5-15 days), intravenous immunoglobulin (6\%) at 6 days (IQR, 2-13 days), tocilizumab (10\%) at 8 days (IQR, 5-13 days), remdesivir (4\%) at 14 days (IQR, 10-15 days), $n$-acetylcysteine (3\%) at 29 days (IQR, 29-30 days), siltuximab $(n=1)$ at 7 days, and anakinra $(n=1)$ at 9 days (Supplemental Table 2). Overall, 15 patients had IL-6 levels drawn and 8 patients received tocilizumab or siltuximab. The median IL-6 level before IL-6-directed therapy was 176.7 pg/ $\mathrm{mL}$ (range, 49.5-1578.4 pg/mL). While patients may have had an inflammatory response similar to hemophagocytic lymphohistiocytosis (HLH), no other HLH-directed treatments were administered. In addition, $48 \%$ of patients received antibacterial coverage for potential superimposed bacterial infection.

Clinical course and outcomes. Forty-four percent of patients required admission, with 24 of 34 admitted on the same day as the positive NPS, while an additional $8 \%$ were already admitted for treatment of their malignancy at the time of positive NPS. Median length of stay for the initial hospitalization was 8 days (IQR, 5-18 days). At last follow-up, 24 patients (71\%) were discharged, with 2 readmitted during the follow-up time. Secondary infections were formally documented in 10 patients (with some having multiple infections) and included bacteremia $(n=3)$, fungal pneumonia $(n$ $=3)$, urinary tract infection $(n=2)$, clostridium difficile diarrhea $(n=2)$, bacterial pneumonia $(n=1)$, and EBV reactivation $(n=1)$. Prophylaxis for venous thrombosis was given in patients with an adequate platelet count ( 24 of 34 ). Two patients developed thromboses, with one having thrombocytopenia precluding anticoagulation and one having a prior history of venous thrombosis; this patient developed a catheter-associated thrombosis when prophylaxis was discontinued due to thrombocytopenia. No cerebrovascular accidents were seen. No prior dialysis-naive patients required dialysis. Fifty-seven percent (44 of 77) of patients did not require supplemental oxygen, while $32 \%$ required a nonrebreather (NRB) or higher level of supplementation (Figure $3 \mathrm{~A})$. Nine patients $(25 \%$ of those admitted) required intubation, with 3 patients extubated, 

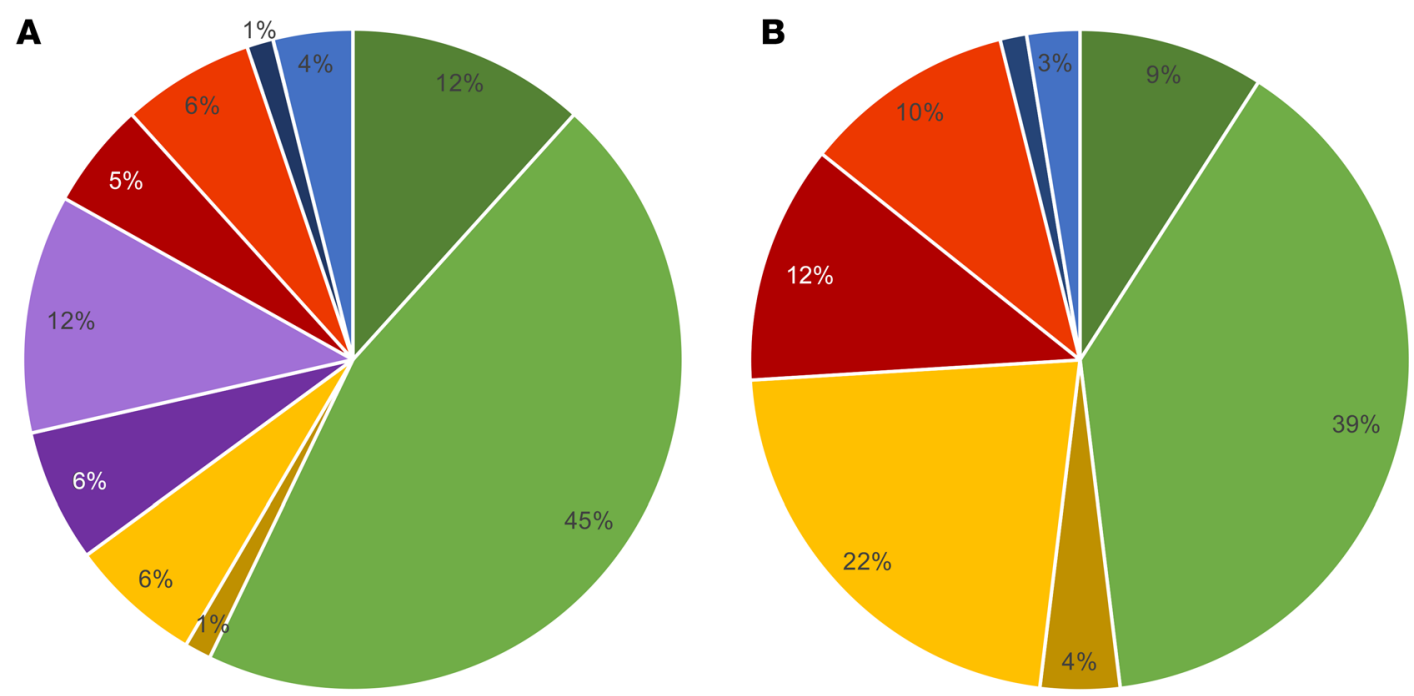

- Room air, active malignancy
- Room air
- Nasal canula, active malignancy
- Nasal canula
- Non-rebreather, active malignancy
- Non-rebreather
- Inutubated, active malignancy
- Intubated
- Unknown, active malignancy

- Mild, active malignancy

- Mild

- Moderate, active malignancy

- Moderate

- Severe, active malignancy

- Severe

- Unknown, active malignancy

- Unknown

Figure 3. Outcomes and disease severity. (A) Highest level of supplemental oxygen given by disease status. (B) COVID-19 disease severity by hematologic malignancy status. Seventy-seven patients (Allo, $n=35$; Auto, $n=37$; CAR T, $n=5$ ). Severity of COVID-19 was defined as mild (no hospitalization required), moderate (hospitalization required), or severe (intensive care unit [ICU] required or goals of care changed to comfort care rather than escalation to the ICU).

5 dying while intubated, and 1 remaining on the ventilator. The median time to extubation or death in the intubated patients was 12 days (IQR, 8-22 days). Ten patients required pressor support in the intensive care unit (ICU). Code status was changed to do not resuscitate on 13 admitted patients, with 5 changed after intubation. Overall, $48 \%, 26 \%$, and $22 \%$ of patients had mild, moderate, or severe COVID-19, with 12 of 17 patients with severe disease dying (Figure 3B). The median time from diagnosis to resolution of symptoms was 14 days (IQR, 10-20 days). Of the 14 patients who died, 8 (57\%) had active disease at COVID-19 diagnosis and 4 were within 1 year of cellular therapy (Figure 4). Overall survival at 30 days was $78 \%$ (95\% CI, 68\%-91\%), with 73\% (CI, 57\%-94\%), $87 \%$ (73\%-100\%), and 60\% (29\%-100\%) of Allo, Auto, and CAR $\mathrm{T}$ recipients alive, respectively (Figure 5).

\section{Factors associated with disease severity}

In an effort to not undercategorize severity, we created a composite endpoint, requiring a NRB or higher oxygen or death at a lower level of oxygen, as there were patients who were not intubated or transferred to the ICU based on goals-of-care discussions with the patient, their family, and the clinical team, taking into account their COVID-19 course and the status of their underlying malignancy. Univariable analysis for this composite endpoint was significant for number of comorbidities (HR for $\geq 2$ vs. 0 comorbidities, 5.41; 95\% CI, 1.84-15.9, $P$ $=0.004)$, presence of infiltrates on initial imaging (HR, 3.08; 95\% CI, 1-9.44, $P=0.032$ ), and neutropenia (HR, 1.15; 95\% CI, 1.02-1.29, $P=0.04$ ) (Table 2). Having more than 2 comorbidities $(P=0.002)$ and an active hematologic malignancy $(P$
$=0.02$ ) predicted for increased disease severity by univariable analysis (Supplemental Table 3).

\section{Evaluation of lymphocyte subsets in patients positive for SARS-CoV-2 after bone marrow transplant}

Monitoring of immune reconstitution after transplant is standard clinical practice at MSKCC, including lymphocyte subsets $\left(\mathrm{CD} 4^{+}\right.$ $\mathrm{T}$ cells, $\mathrm{CD} 8^{+} \mathrm{T}$ cells, $\mathrm{CD} 19^{+} \mathrm{B}$ cells, $\mathrm{CD} 56^{+} \mathrm{CD} 16^{+} \mathrm{NK}$ cells, and $\mathrm{CD}^{+} \mathrm{CD} 56^{+} \mathrm{CD} 16^{+} \mathrm{NKT}$ cells) and, in some patients, additional $\mathrm{T}$ cell populations, including naive $\left(\mathrm{CD} 45 \mathrm{RA}^{+} \mathrm{CCR} 7^{+}\right)$, central memory $\left(\mathrm{CD}^{2} 5 \mathrm{RA}^{-} \mathrm{CCR} 7^{+}\right)$, effector memory (CD45RA-CCR7 $\left.{ }^{-}\right)$, and effector memory CD45RA ${ }^{+}$or TEMRA cells $\left(\mathrm{CD}^{2} 5 \mathrm{RA}^{+} \mathrm{CCR} 7^{-}\right)$ $(14,15)$. During the study period, immune subset analyses were performed in 32 of 77 patients, including 17 Allo, 12 Auto, and 3 CAR T recipients. We selected 25 patients (including 1 patient with presumed COVID-19) within 1 week of any positive SARS-CoV-2 PCR test for further analysis (Table 3 and Supplemental Figure 1).

Infection with SARS-CoV-2 is related to a reduction in lymphocyte populations. Because transplant patients are a uniquely heterogeneous population with regards to the circulating immune cells affected by the type of transplant, state of immune reconstitution, immunosuppression regimen, GVHD, and disease status, we used immunologic profiling before COVID-19 diagnosis available in 12 of 25 patients as an internal control. Consistent with findings seen in prior studies, a reduction from the pre-COVID-19 baseline in ALC was observed in this cohort, except for in 1 patient, whose prior immune subsets were performed just after completing conditioning for CAR T therapy. The reduction in lymphocytes affected all subsets for most patients, particularly CD4 and CD8 T cells; in 


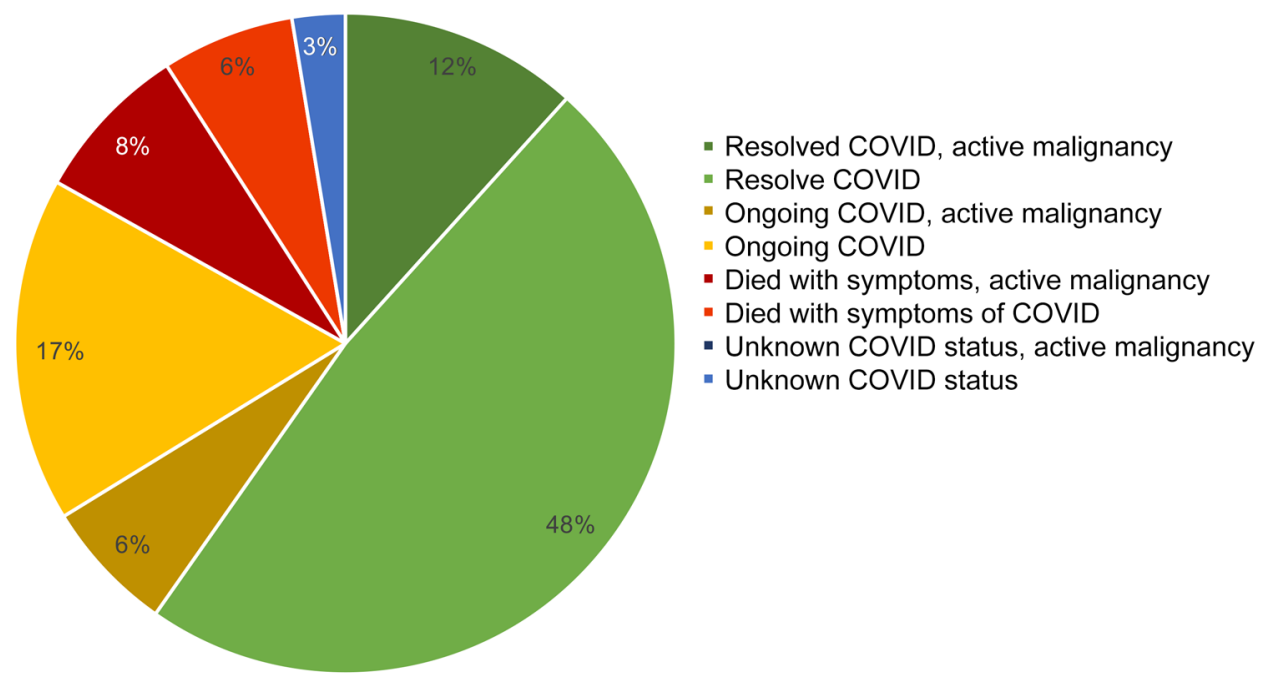

Figure 4. COVID-19 status at last contact. Seventy-seven patients (Allo, $n=35$; Auto, $n=37$; CAR T, $n=5$ ). COVID-19 was defined as resolved at the end of clinical symptoms.

some patients B cells and NK cells remained stable or increased slightly (Figure 6A). The CD4/CD8 ratio varied widely across patients with a trend toward a relative increase in CD4 $\mathrm{T}$ cells (Supplemental Figure 2A). For Allo recipients within 2 years after transplant, we further compared lymphocyte subset data with the expected after HCT immunologic reconstitution from available historical control cohorts (Figure 6B and Supplemental Figure 3), highlighting how COVID-19 is associated with lower lymphocyte counts, particularly in the T cell compartment.

Detailed $\mathrm{T}$ cell phenotyping available in 18 of 25 patients with COVID-19 revealed that CD4 cells were predominantly effector memory cells while CD8 cells had a TEMRA phenotype $\left(\mathrm{CCR}^{-} \mathrm{CD} 45 \mathrm{RA}^{+}\right)$; naive cells were similar in both CD4 and CD8 cells (Supplemental Figure 4). Six patients had prior T cell subset profiling data available within 1 year of COVID-19 (Supplemental Figure 5). There was a trend toward an increase in percentage of $\mathrm{CD}^{+}$, but not $\mathrm{CD}^{+}$, TEMRA cells during the COVID-19 window; however, this was not seen by absolute counts.

Patients can develop IgG antibody responses to SARS-COV-2 despite lymphopenia. During the time of our study, a SARS-CoV-2 antibody test became available. Thirty-eight patients (49\%) had antibody testing done at a median of 37 days after diagnosis (IQR, $28-48$ ) with $66 \%$ of those developing antibodies, including 5 of 10 patients on immunosuppressive medications. In 7 patients who received convalescent plasma, antibody testing at least 2 weeks after infusion was negative in 6 . For the patient with antibodies, repeat testing 1 week later remained positive and is thought to be a true positive response. In a subset of 8 patients who had not received convalescent plasma, but had a positive antibody and immune profiling performed within the COVID-19 period, 6 patients had circulating absolute B cells counts under 100 cells/ $\mu \mathrm{L}$, including 2 with no detectable circulating B cells but measurable IgG levels (Supplemental Figure 6).

Lymphopenia with COVID-19 does not appear to impair immune reconstitution in all patients that had received bone marrow transplant. We next sought to investigate the persistence of lymphopenia associated with COVID-19. Figure 7 illustrates the trajectory lymphocyte populations before, during, and in recovery from COVID-19 in a patient with acute myeloid leukemia disease, who received a trans- plant from a haploidentical donor, highlighting how lymphocytes began to recover, even though the patient did not yet have a detectable SARS-CoV-2 antibody. Available data from other patients had an overall similar trajectory, aside from 1 patient with a fatal infection combined with underlying MDS (Supplemental Figure 7).

\section{Discussion}

Here, we present the largest series of COVID-19 outcomes for patients who have received cellular therapies, including Allo, Auto, and CAR T. The percentage of patients with underlying comorbidities is similar to what would be expected after transplant (16). Overall, almost half of the patients were monitored and recovered entirely as outpatients without any outpatient deaths. Treatments varied throughout the time period due to rapid iterative changes in clinical management algorithms. Documented secondary infections were uncommon, including in those patients who received IL-6-directed therapies, similar to CAR T recipients treated with tocilizumab for cytokine release syndrome $(17,18)$. Interestingly,

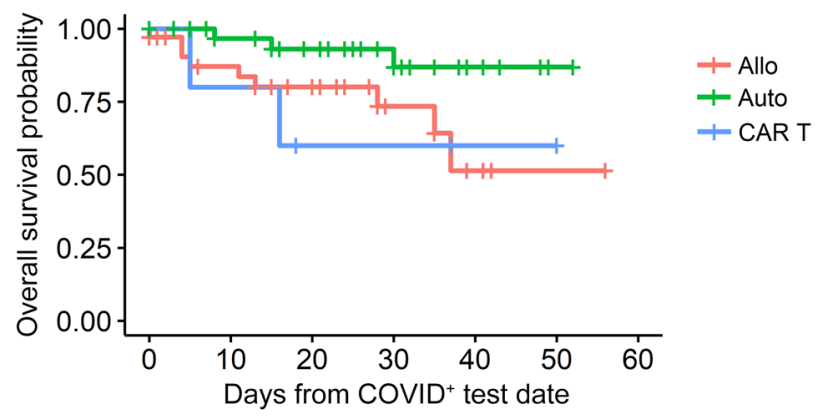

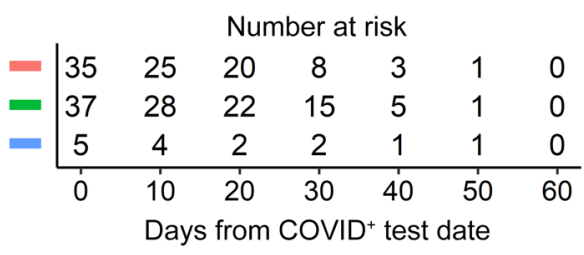

Figure 5. Overall survival by cell therapy type. Seventy-seven patients (Allo, $n=35$; Auto, $n=37$; CAR T, $n=5$ ). 
Table 2. Univariable analysis of composite endpoint of requiring nonrebreather or more oxygen and death at lower level of oxygen

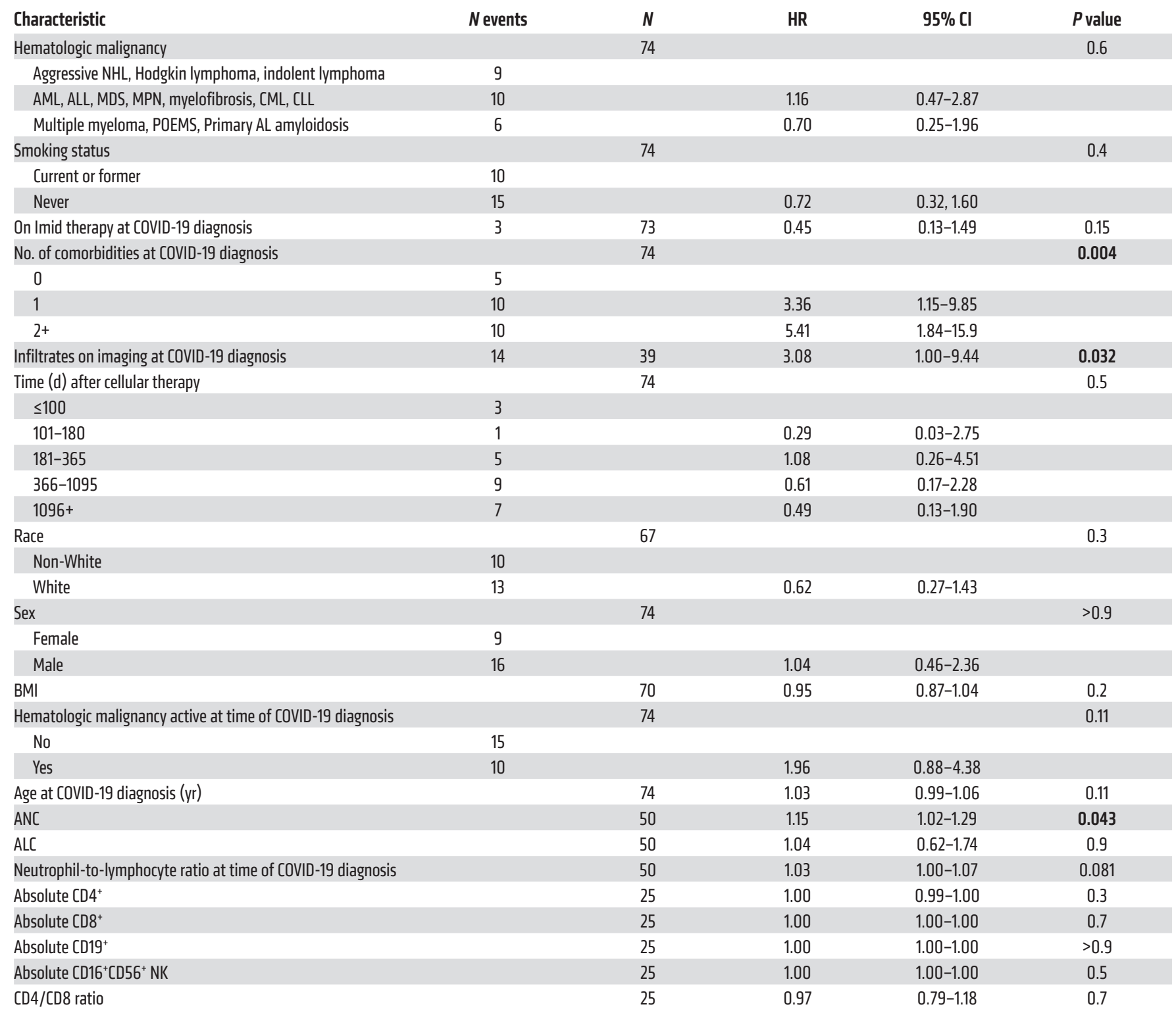

$N$ events are not shown for continuous variables. Significant values are shown in bold. Univariable associations between clinical characteristics and COVID-19 severity were assessed using the Kruskal-Wallis test, $\chi^{2}$ test of independence, and Fisher's exact test, as appropriate. NHL, non-Hodgkin Iymphoma; AML, acute myeloid leukemia; ALL, acute lymphoblastic leukemia; MDS, myelodysplastic syndrome; MPN, myeloproliferative neoplasm; CML, chronic myeloid leukemia; CLL, chronic lymphocytic leukemia; POEMS, polyneuropathy, organomegaly, endocrinopathy, M protein, and skin changes; ANC, absolute neutrophil count; ALC, absolute lymphocyte count; Imid, immunomodulatory agent (e.g., lenalidomide, pomalidomide).

time from cellular therapy and many previously reported risk factors for disease severity were not significant in our analysis, though analyses were limited by the small number of events. Immune alterations, most predominantly lymphopenia, were seen, but it appeared that improvements in lymphocyte counts occurred within a short period of time after resolution of symptoms.

The clinical presentation and overall course of COVID-19 was similar to those from other large cohorts from academic centers in New York $(19,20)$; patients with cancer $(2,21-23)$, particularly those with hematologic malignancies $(7,24-27)$; and patients after solid-organ transplant on immunosuppression (28). Symptoms at presentation were common across all cohorts and included fevers, cough, and shortness of breath. The presence of infiltrates the at time of diagnosis and requirement for oxygen supplementation portended worse outcomes. In a cohort of solid-organ transplant patients treated in the New York Presbyterian system, the distribution of disease severity appears to be increased when on immunosuppression (28) compared with our population (majority not on immunosuppression), with $24 \%, 46 \%$, and $30 \%$ versus $48 \%$, $26 \%$, and $22 \%$ of patients having mild, moderate, and severe dis- 
Table 3. Immune profiling correlates of patients with COVID-19

\begin{tabular}{lcccccccc} 
Characteristic & \multicolumn{2}{c}{ Overall } & \multicolumn{2}{c}{ Allo } & \multicolumn{2}{c}{ Auto } & \multicolumn{2}{c}{ CAR T } \\
& $\mathbf{N}$ & Median (IQR) & $\mathbf{N}$ & Median (IQR) & $\boldsymbol{N}$ & Median (IQR) & $\boldsymbol{N}$ & Median (IQR) \\
Absolute CD3 $^{+}$ & 25 & $354(119-636)$ & 12 & $365.5(54.5-526)$ & 10 & $376.5(212.8-804)$ & 3 & $354(216-611.5)$ \\
Absolute CD4 $^{+}$ & 25 & $140(51-194)$ & 12 & $146.5(28.8-202.8)$ & 10 & $154(119-199.2)$ & 3 & $51(39-73.5)$ \\
Absolute CD8 $^{+}$ & 25 & $221(35-327)$ & 12 & $180(26-283.5)$ & 10 & $212.5(48.5-614.5)$ & 3 & $254(140-507)$ \\
Absolute CD19+ $^{+}$ & 25 & $11(0-50)$ & 12 & $9(0.8-26.8)$ & 10 & $49.5(5.2-80)$ & 3 & $0(0-0)$ \\
Absolute NK & 25 & $100(52-151)$ & 12 & $115.5(92.2-252.2)$ & 10 & $56(38.5-116.5)$ & 3 & $72(54-98.5)$ \\
CD4/CD8 ratio & 25 & $0.9(0.5-1.6)$ & 12 & $1(0.6-2.1)$ & 10 & $0.7(0.4-1.4)$ & 3 & $0.4(0.2-1.2)$
\end{tabular}

Allo, allogeneic hematopoietic stem cell transplantation; Auto, autologous hematopoietic stem cell transplantation; CAR T, CD19-directed chimeric antigen receptor T cell therapy. subset but rather leads to marked reduction across lymphocyte populations (10-13). Phenotypic evaluation of 20 patients without HCT who recovered revealed a slight increase in the percentage of CD3 $\mathrm{T}$ cells with a reduction in CD19 B cells compared with healthy controls (34); however, pre-COVID-19 or mid-COVID-19 lymphocyte characterization was not available. A strength of ease, respectively. While our median follow-up was 23 days, the IQR for resolution of symptoms was 10-20 days, and there were few patients with ongoing symptoms at the time of our data cutoff.

In our cohort, the overall mortality rate was $41 \%$ in hospitalized patients, but this was largely driven by patients with active malignancy, especially relapsed leukemia, in whom the goals of care were affected both by COVID-19 severity and the decision to forgo anticancer treatment during an active infection. For the patients with hematologic malignancies treated in the Montefiore Health system, the case fatality rate was $37 \%$ (20 of 54) (22). In our cohort of cellular therapy recipients without active malignancy, the death rate was $21 \%$, which matched the reported mortality of patients hospitalized with COVID-19 in New York (20).

Interestingly, outcomes in our study were not different based on the type of hematologic malignancy. A large portion of patients had Auto for multiple myeloma, and our results are similar to those in the Mount Sinai cohort, in which 22 of 54 (41\%) had an Auto previously (24). Exposure to a person infected with SARS-CoV-2 was a significant risk factor for developing COVID-19 in a cohort of chronic myeloid leukemia patients treated with tyrosine kinase inhibitors (26). In our study, $45 \%$ of patients had a known exposure outside of the medical system. Therefore, while limited clinic visits and telemedicine interactions with the medical system are important, social distancing, use of personal protective equipment, and infection control, even at home, may be needed to protect patients with hematologic malignancies from contracting SARS-CoV-2 and presents an obstacle to address during a potential second wave.

GI symptoms in COVID-19 present a particular challenge in Allo recipients because they may be difficult to differentiate from GVHD. In our cohort, for those patients on immunosuppression, their GVHD did not worsen. Importantly, though we would be concerned for an infection triggering GVHD, no Allo recipients had new GVHD arise during their COVID-19 course, with the caveat of a relatively short follow-up window.

Understanding the adaptive immune response in COVID-19 bone marrow transplantation (BMT) recipients is critical because of the immunocompromised nature of these patients and the well-established role of viral infection in modulating immune reconstitution following transplantation (29-33). Lymphopenia is a common feature of SARS-CoV-2 infection, particularly in severe cases. Our data are consistent with that of others in identifying that SARS-CoV-2 infection does not specifically target an immune our study is that we were able to compare longitudinal immune subsets before and after SARS-CoV-2 infection. We demonstrate that although some of our patients were less than one year after transplant they indeed were able to begin to recover T cells. Furthermore, despite marked lymphopenia, including a lack of circulating B cells, several patients were able to mount a SARS-CoV-2 antibody, suggesting antibody production from noncirculating lymph node or tissue-resident cells. The level and durability of this response remain uncertain. A similar experience has been reported in patients with multiple myeloma treated at Mount Sinai Health System, who also developed an antibody response (24). For patients who received lymphocyte-depleting chemotherapy or cellular therapies, elucidating lymphocyte requirements for adequate immunologic control of the infection will be fundamental for developing clinical guidelines. Given that some many transplant patients may have impaired humoral immunity due to prior treatment history and/or cellular therapy, we predict that serologic conversation in the transplant population will be lower than that of the general population.

Consistent with published data $(10,11)$, detailed T cell analyses suggest an increase in CD8 TEMRA cells during SARS-CoV-2 infection, an indication of a terminally differentiated phenotype (35). Early data suggest an exhausted phenotype in CD8 T cells in patients with SARS-CoV-2 infection $(10,11)$, which may reflect an active viral infection but may also be part of the picture of why some patients with SARS-CoV-2 infection are unable to mount an adequate antiviral response (8). We did not detect a clear association with degree of lymphopenia and disease severity, as has been shown previously (12), but this may be a reflection of our small sample size combined with the immunologically complex nature of our population following cellular therapies. We also acknowledge that the neutrophil-to-lymphocyte ratio may be affected by a diversity of medical conditions, including active hematologic malignancy, and may not be as informative in this population as compared with the general public.

Potential limitations of the interpretation of immunologic subsets in our patients include population heterogeneity, including a diversity of graft sources and distinct immunosuppression regimens, combined with confounding clinical variables, such as CMV reactivation, GVHD, and disease relapse. As a result, we focused our analyses on trends before and after COVID-19 within the same patient and sought to contextualize our findings with 
A

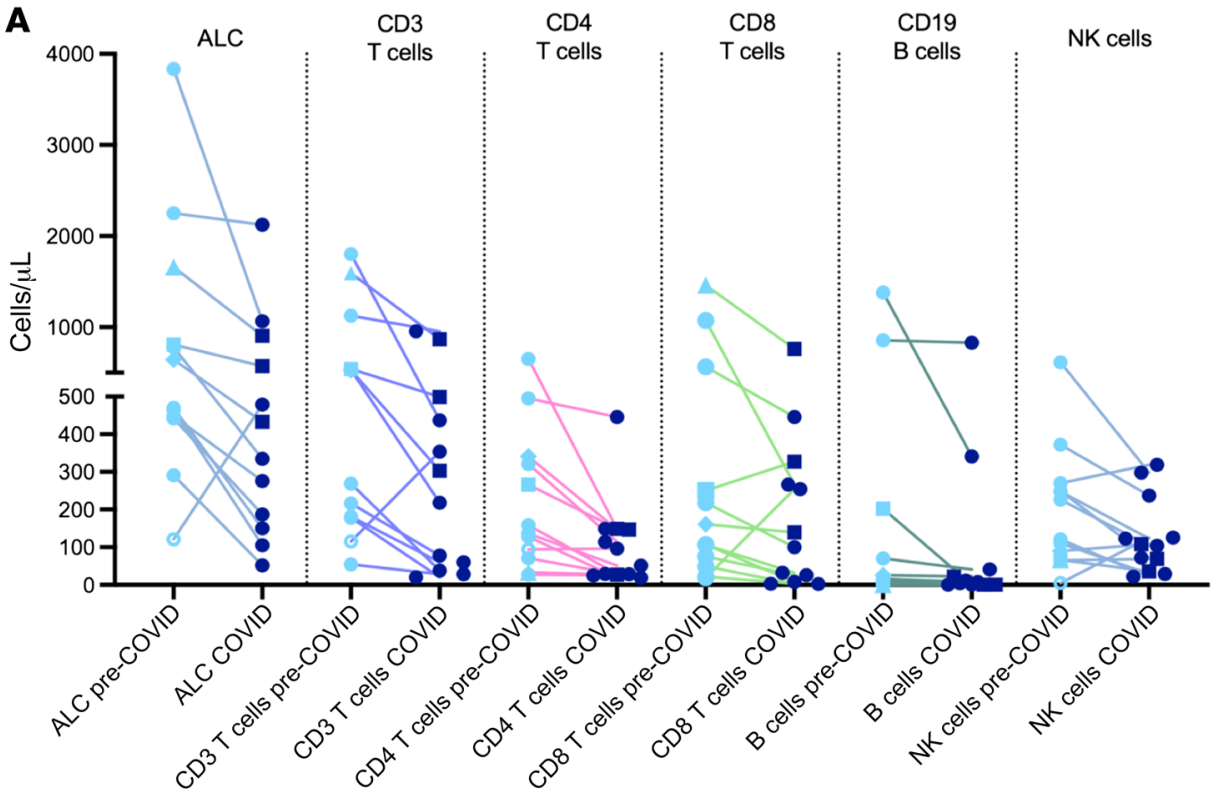

Day 0 CAR-T $\triangle 8$ days prior to CAR-T Active disease $>$ Minimal residual disease
B
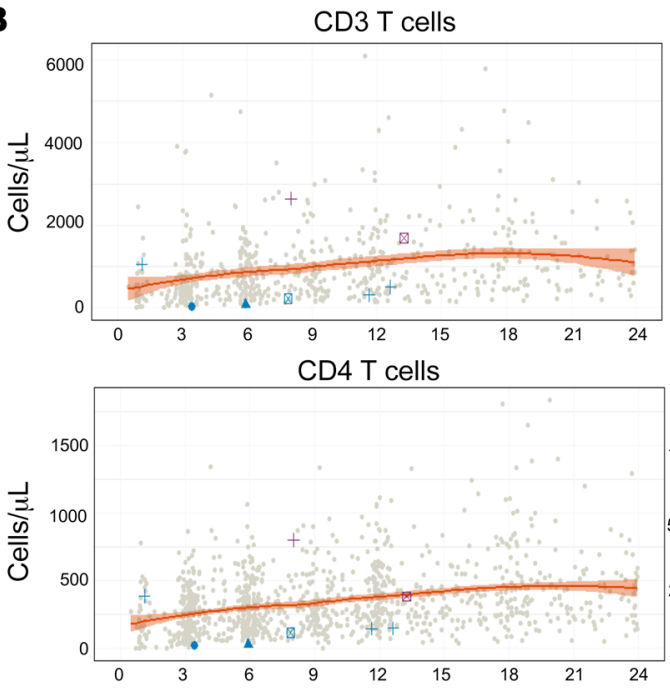

CD3-CD56+, CD16 ${ }^{+} \mathrm{NK}$ cells

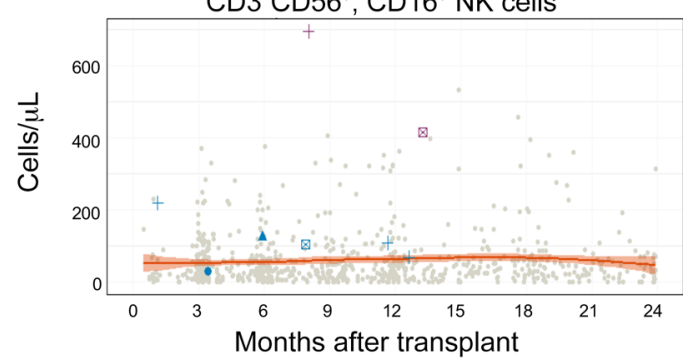

CD19 B cells
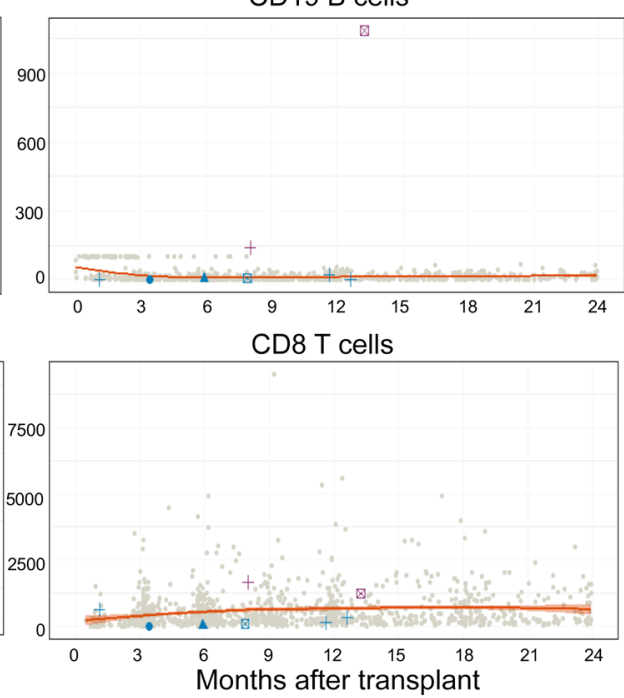

PCR timing

-PCR within a week of immune subsets

- PCR beyond a week of immune subsets

Allograft

+ Unmodified matched PBSC

\Unmodified mismatched unrelated PBSC

- Haploidentical, bone marrow

$\Delta$ Haploidentical, bone marrow available data from historical controls, recognizing that a much larger cohort is needed to fully characterize risk factors for disease severity. The decision to require swabbing within 1 week of immune profiling was an arbitrary cutoff. However, because of the wide range of COVID-19 symptoms, a positive PCR test, even if late into a patient's course, was an objective measurement of recent active infection. An area of active research is incorporating the Ct of the PCR to infer presence of viable virus (36).
Other limitations include lack of laboratory studies, including immunophenotyping, or diagnostic imaging in patients who had milder disease, as these patients were able to continue to follow state and federal recommendations and were appropriately advised to remain isolated and to avoid nonurgent visits to the health care setting. The patients in this study were diagnosed during the initial surge in New York City, and, as such, testing and treatment were based on the available data and safety guide- 


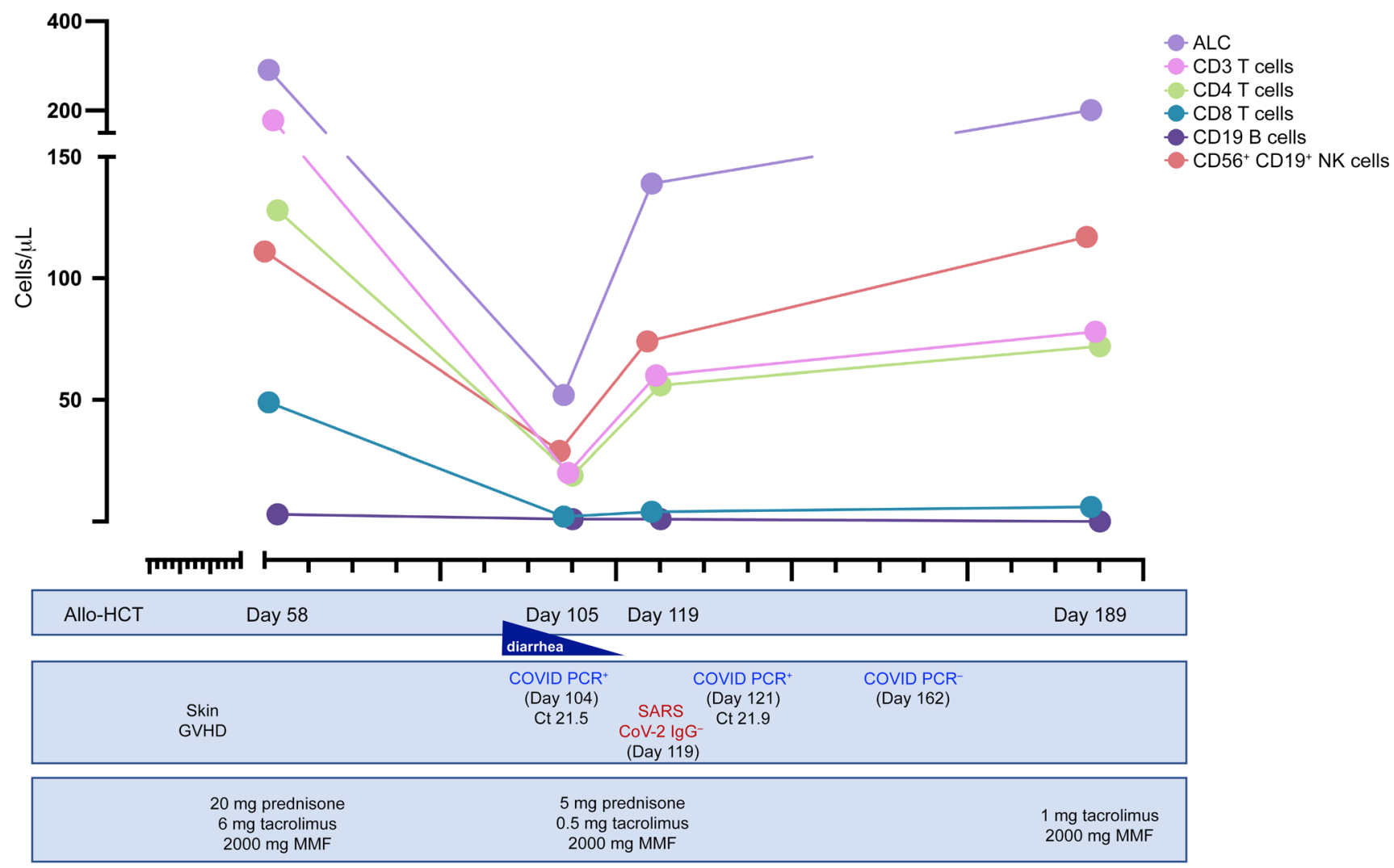

Figure 7. Tracking lymphocyte subsets over time before, during, and in recovery from a COVID-19 infection. Allo recipient who received a haploidentical transplant for acute myeloid leukemia. Available COVID-19 PCR data with cycle threshold (Ct) and antibody status included.

lines of the time. Patients were identified for inclusion by positive PCR testing. As a result, additional symptomatic patients with COVID-19 may not have been included due to negative testing based on timing or sensitivity of the test. Asymptomatic patients may also have been missed, as they were only tested prior to a needed procedure early during the pandemic when resources were more constrained. We acknowledge that diagnostic work-up and treatment in nonsurge conditions and data obtained over time may change observed outcomes as further cases are diagnosed. As the median time from cell therapy to COVID-19 diagnosis was 782 days, the results may not be generalizable to the course of patients early after infusion. Furthermore, in the absence of systemic testing, we cannot assess a potential association between active malignancy and a higher likelihood of having COVID-19. Nevertheless, as we have included all of the patients who tested positive by PCR at our center, we are able to compare the outcomes of those who did and did not have active malignancy at the time of their COVID-19 diagnosis. Finally, given the limited sample size and event rates, only univariable associations could be explored as multivariable modeling was infeasible.

The American Society for Transplant and Cellular Therapy, the European Society for Blood and Marrow Transplantation (EBMT), the Worldwide Network for Blood and Marrow Transplantation, and the Center for International Blood and Marrow Transplant Research (CIBMTR) continue to update guidelines for the treatment of COVID-19 in this population (37-40). Furthermore CIBMTR and EBMT continue to collect cases for multicenter analyses to improve outcomes for cellular therapy patients $(41,42)$. Important issues include addressing those with persistently positive NPS and the question of shedding of residual viral RNA versus infectious actively replicating virus $(36,43,44)$. Some patients were not able to clear their NPS, given that they are immunocompromised, and the $\mathrm{Ct}$ value cutoff for safety and ability to resume treatment or discontinue precautions for cellular therapy patients is an active area of investigation at MSKCC.

In conclusion, patients who have received cellular therapies, including Allo, Auto, and CAR T therapy, were able to recover from COVID-19 infection and mount an antibody response, with similar overall survival to the general hospitalized population. Poor outcomes were more frequently seen in those with active relapsed disease and with risk factors akin to their noncancer counterparts, such as comorbidities and neutropenia. Given the potential for prolonging survival and potential cure, it remains critical to safely continue treating patients with cellular therapies during the global pandemic and to determine successful interventions for those early after cellular therapy who remain immunocompromised.

\section{Methods}

Patients who received Allo, Auto, or CAR T therapy were identified from the MSKCC institutional database. Patients were included if they had a positive NPS for SARS-CoV-2 either at MSKCC or through the MSKCC exchange system, connecting our electronic records to electronic records at select institutions. Presumed positive patients were 
defined as having common COVID-19 symptoms with either a known exposure or imaging consistent with COVID-19.

The electronic medical record and institutional databases were abstracted for demographic information and medical history, including comorbidities, treatment characteristics, and the presence and treatment of GVHD. For patients who underwent testing at outside locations, additional information and records were abstracted as available. Laboratory and radiology information at the time of SARS-CoV-2 testing and subsequent admission (if admitted) as well as COVID-19specific treatments, complications, and outcomes were collected from March 11, 2020, through May 12, 2020. Follow-up SARS-CoV-2 testing was included through June 2, 2020. Severity of COVID-19 was defined as mild (no hospitalization required), moderate (hospitalization required), and severe (ICU required or goals of care changed to comfort care rather than escalation to the ICU). COVID-19 was considered resolved once clinical symptoms were no longer present.

Immunophenotyping of peripheral blood mononuclear cells via flow cytometry was performed in the MSKCC clinical laboratory. The lymphocyte panel included CD45 FITC (BD, 340664, clone 2D1), CD56+16 PE (BD 340705, clone B73.1; BD 340724, clone NCAM 16.2), CD4 PerCP-Cy5.5 (BD 341653, clone SK3), CD45RA PC7 (BD 649457, clone L48), CD19 APC (BD 340722, clone SJ25C1), CD8 APC-H7 (BD 641409, clone SK1), and CD3 BV 421 (BD 562426, clone UCHT1). The naive/effector T panel included CD45 FITC (BD 340664, clone 2D1), CCR7 PE (BD 560765, clone 150503), CD4 PerCP-Cy5.5 (BD 341653, clone SK3), CD38 APC (BioLegend, 303510, clone HIT2), HLA-DR V500 (BD 561224, clone G46-6), CD45RA PC7 (BD 649457, clone L48), CD8 APC-H7 (BD 641409, clone SK1), and CD3 BV 421 (BD 562426, clone UCHT1). Lymphocyte populations were tracked over time and compared with historical control data for patients previously studied at MSKCC within 2 years after transplant $(14,45)$.

At MSKCC, NPS samples were collected using flocked swabs (Copan Diagnostics) and placed in viral transport media. SARS-CoV-2 RNA was detected using the CDC protocol, targeting 2 regions of the nucleocapsid gene (N1 and N2), with the following modifications. Nucleic acids were extracted from NPS samples using the NUCLISENS EasyMag (bioMérieux) following an off-board, pre-lysis step. Real-time reverse-transcription PCR was performed on the ABI 7500 Fast (Applied Biosystems) in a final reaction volume of $20 \mu \mathrm{L}$, including of $5 \mu \mathrm{L}$ extracted nucleic acids. Samples were reported as positive if both the N1 and N2 targets were detected (Ct of less than 40, with a maximum of 45 cycles run). Cts in patients with serial NPS were evaluated to explore the relationship between clinical outcomes and viral load. Serum or plasma was analyzed on the Abbott Architect i2000 analyzer in an automated 2-step immunoassay for the qualitative detection of IgG antibodies against the nucleocapsid protein of SARS-
CoV-2 using chemiluminescent microparticle immunoassay technology. $\mathrm{N}$-acetylcysteine treatment was given on a clinical trial (Clinicaltrials.gov NCT04374461), while convalescent plasma (Clinicaltrials.gov NCT04338360) and remdesivir (Clinicaltrials.gov NCT04323761) were given through expanded access programs.

Statistics. Descriptive statistics were used to summarize patient characteristics, lab values, and disease characteristics. Overall survival from the date of COVID-19 diagnosis to death or last contact date was estimated using Kaplan-Meier methodology. Univariable associations between clinical characteristics and a composite endpoint of requiring a NRB or higher amount of oxygen and death was analyzed using Cox models, where time was defined from the date of COVID-19 diagnosis. Univariable associations between clinical characteristics and COVID-19 severity were assessed using the Kruskal-Wallis test, $\chi^{2}$ test of independence, and Fisher's exact test, as appropriate. Both sets of univariable analyses were performed among patients with labs performed within 1 week of COVID-19 diagnosis. $P$ values of less than 0.05 were considered significant.

Study approval. This study was approved by the Institutional Review Board of MSKCC.

\section{Author contributions}

GLS, SD, GP, RT, CSS, and MAP designed the study. GLS, SD, YJL, RT, PBD, JR, NEB, TJ, CG, PM, MK, and LR acquired the data. GLS, SD, JAL, SMD, PM, MK, and MAP analyzed the data. CC, JUP, IP, MS, SV, AD, TMH, MK, EP, and GP cared for the patients included in this study. GLS, SD, and MAP wrote the manuscript, and YJL, RT, PBD, JAL, JR, SMD, CC, JUP, IP, MS, NEB, TJ, SV, AD, CSS, JNB, SAG, CG, PM, TMH, MK, LR, MRMVDB, EP, and GP critically reviewed the manuscript. GLS and SD contributed equally to this work. GLS is listed first as she initiated the project.

\section{Acknowledgments}

We thank Jedd Wolchok for guidance and support for this project. This research was supported in part by NIH award P01 CA23766 and NIH/National Cancer Institute Cancer Center Support grant P30 CA008748. The content is solely the responsibility of the authors and does not necessarily represent the official views of the NIH. The authors thank Theodore and Laura Hromadka for their support. SD was supported in part by Clinical Scholars 2T32 CA009512 through Memorial Sloan Kettering internal funding.

Address correspondence to: Gunjan L. Shah, Memorial Sloan Kettering Cancer Center, 1275 York Avenue, New York, New York 10065, USA. Phone: 212.639.2000; Email: shahg@mskcc.org
1. Johns Hopkins Coronavirus Resource Center. COVID-19 United States Cases by County. https:// coronavirus.jhu.edu/us-map. Accessed Jun 2, 2020.

2. Dai M, et al. Patients with cancer appear more vulnerable to SARS-CoV-2: a multicenter study during the COVID-19 outbreak. Cancer Discov. 2020;10(6):783-791.

3. Nicholson B, et al. NPI-2358 is a tubulindepolymerizing agent: in-vitro evidence for activity as a tumor vascular-disrupting agent.
Anticancer Drugs. 2006;17(1):25-31.

4. Singh AV, et al. A novel vascular disrupting agent plinabulin triggers JNK-mediated apoptosis and inhibits angiogenesis in multiple myeloma cells. Blood. 2011;117(21):5692-5700.

5. Mita M, et al. Phase 1 first-in-human trial of the vascular disrupting agent plinabulin(NPI-2358) in patients with solid tumors or lymphomas. Clin Cancer Res. 2010;16(23):5892-5899.

6. Mark T, et al. Atypical serum immunofixation patterns frequently emerge in immunomodulato- ry therapy and are associated with a high degree of response in multiple myeloma. Br J Haematol. 2008;143(5):654-660.

7. Hultcrantz M, et al. COVID-19 infections and outcomes in patients with multiple myeloma in New York City: a cohort study from five academic centers [published online September 15, 2020]. Blood Can Discov. https://doi. org/10.1158/2643-3230.BCD-20-0102.

8. Vardhana SA, Wolchok JD. The many faces of the anti-COVID immune response.J Exp Med. 
2020;217(6):e20200678.

9. Li J, et al. Clinical features of familial clustering in patients infected with 2019 novel coronavirus in Wuhan, China. Virus Res. 2020;286:198043.

10. Mazzoni A, et al. Impaired immune cell cytotoxicity in severe COVID-19 is IL-6 dependent. JClin Invest. 2020;130(9):4694-4703.

11. Zheng M, et al. Functional exhaustion of antiviral lymphocytes in COVID-19 patients. Cell Mol Immunol. 2020;17(5):533-535.

12. Chen $\mathrm{G}$, et al. Clinical and immunological features of severe and moderate coronavirus disease 2019. J Clin Invest. 2020;130(5):2620-2629.

13. Qin C, et al. Dysregulation of immune response in patients with coronavirus 2019 (COVID-19) in Wuhan, China. Clin Infect Dis. 2020;71(15):762-768.

14. Jain $\mathrm{T}$, et al. Cytomegalovirus reactivation promotes $\mathrm{CD} 8+\mathrm{T}$ cell subset recovery after unmodified allogeneic hematopoietic cell transplantation. Biol Blood Marrow Transplant. 2019;25(3):S326-S327.

15. Politikos I, et al. Robust CD4+ T-cell recovery in adults transplanted with cord blood and no antithymocyte globulin. Blood Adv. 2020;4(1):191-202.

16. Savani BN, Griffith ML, Jagasia S, Lee SJ. How I treat late effects in adults after allogeneic stem cell transplantation. Blood. 2011;117(11):3002-3009.

17. Wudhikarn $\mathrm{K}$, et al. Infection during the first year in patients treated with CD19 CAR T cells for diffuse large B cell lymphoma. Blood Cancer J. 2020;10(8):79.

18. Frigault MJ, et al. Tocilizumab not associated with increased infection risk after CAR T-cell therapy: implications for COVID-19? Blood. 2020;136;(1):137-139.

19. Petrilli CM, et al. Factors associated with hospital admission and critical illness among 5279 people with coronavirus disease 2019 in New York City: prospective cohort study. BMJ. 2020;369:m1966.

20. Richardson $S$, et al. Presenting characteristics, comorbidities, and outcomes among 5700 patients hospitalized with COVID-19 in the New York City area. JAMA. 2020;323(20):2052-2059

21. Liang $\mathrm{W}$, et al. Cancer patients in SARS-CoV-2 infection: a nationwide analysis in China. Lancet Oncol. 2020;21(3):335-337.
22. Mehta V, et al. Case fatality rate of cancer patients with COVID-19 in a New York hospital system. Cancer Discov. 2020;10(7):935-941.

23. Robilotti EV, et al. Determinants of COVID-19 disease severity in patients with cancer. Nat Med. 2020;26:1218-1223.

24. Wang B, et al. A tertiary center experience of multiple myeloma patients with COVID-19: lessons learned and the path forward. J Hematol Oncol. 2020;13(1):94.

25. Malard F, et al. COVID-19 outcomes in patients with hematologic disease [published online May 6, 2020]. Bone Marrow Transplant. https://doi. org/10.1038/s41409.020.0931-4.

26. Li W, et al. COVID-19 in persons with chronic myeloid leukaemia. Leukemia. 2020;34(7):1799-1804

27. Shah V, et al. Poor outcome and prolonged persistence of SARS-CoV-2 RNA in COVID-19 patients with haematological malignancies; King's College Hospital experience. Br J Haematol. 2020;190(5):e279-e282.

28. Pereira MR, et al. COVID-19 in solid organ transplant recipients: initial report from the US epicenter. Am J Transpl. 2020;20(7):1800-1808.

29. van Heijst JWJ, et al. Quantitative assessment of T cell repertoire recovery after hematopoietic stem cell transplantation. Nat Med.2013;19(3):372-377.

30. Muraro PA, et al. T cell repertoire following autologous stem cell transplantation for multiple sclerosis. J Clin Invest. 2014;124(3):1168-1172.

31. de Koning C, Admiraal R, Nierkens S, Boelens JJ. Human herpesvirus 6 viremia affects T-cell reconstitution after allogeneic hematopoietic stem cell transplantation. Blood Adv. 2018;2(4):428-432.

32. Kanakry CG, et al. Origin and evolution of the $T$ cell repertoire after posttransplantation cyclophosphamide. JCI Insight. 2016;1(5):e86252.

33. Suessmuth Y, et al. CMV reactivation drives posttransplant T-cell reconstitution and results in defects in the underlying TCR $\beta$ repertoire. Blood. 2015;125(25):3835-3850.

34. Grifoni A, et al. Targets of $\mathrm{T}$ cell responses to SARS-CoV-2 coronavirus in humans with COVID-19 disease and unexposed individuals. Cell.2020;181(7):1489-1501.e15
35. Thome JJC, et al. Spatial map of human T cell compartmentalization and maintenance over decades of life. Cell. 2014;159(4):814-828.

36. Bullard J, et al. Predicting infectious SARS-CoV-2 from diagnostic samples [published online ahead of print May 22, 2020]. Clin Infect Dis. https:// doi.org/10.1093/cid/ciaa638.

37. Barlogie $\mathrm{B}$, et al. Long-term outcome results of the first tandem autotransplant trial for multiple myeloma. Br J Haematol. 2006;135(2):158-164.

38. Hahn TE, et al. Minimal residual disease (MRD) assessment before and after autologous hematopoietic cell transplantation (AutoHCT) and maintenance for multiple myeloma (MM): results of the Prognostic Immunophenotyping for Myeloma Response (PRIMeR) study. Biol Blood Marrow Transplant. 2019;25(3):S4-S6.

39. Bachanova $\mathrm{V}$, et al. Chimeric antigen receptor $\mathrm{T}$ cell therapy during the COVID-19 pandemic. Biol Blood Marrow Transplant. 2020;26(7):1239-1246.

40. Algwaiz G, et al. Real-world issues and potential solutions in HCT during the COVID-19 pandemic: perspectives from the WBMT and the CIBMTR's Health Services and International Studies Committee [published online July 24, 2020]. Biol Blood Marrow Transplant. https://doi. org/10.1016/j.bbmt.2020.07.021.

41. CIBMTR. Covid Reports. http://www.cibmtr.org/ Covid19/Pages/Covid-Reports.aspx. Accessed June 8, 2020 .

42. Ljungman P. COVID-19 in stem cell transplant patients. What do we know? EBMT Webinar. March 30, 2020. https://www.ebmt.org/sites/ default/files/2020-03/EBMT Webinar COVID19 in stem cell transplant patients 20.03.2020. pdf. Accessed June 2, 2020.

43. He X, et al. Temporal dynamics in viral shedding and transmissibility of COVID-19. Nat Med. 2020;26(5):672-675.

44. Yu F, et al. Quantitative detection and viral load analysis of SARS-CoV-2 in infected patients. Clin Infect Dis. 2020;71(15):793-798.

45. Politikos I, et al. Angiogenic factors correlate with $\mathrm{t}$ cell immune reconstitution and clinical outcomes after double-unit umbilical cord blood transplantation in adults. Biol Blood Marrow Transplant. 2017;23(1):103-112. 